\title{
El aula virtual: una experiencia educativa desde diversos ámbitos universitarios latinoamericanos
}

\section{The virtual classroom: an educational experience from various Latin American university fields}

\author{
Irma Abrigo-Córdova \\ irmabrigocordova2016@gmail.com \\ Universidad Nacional de Loja \\ Ecuador \\ https://orcid.org/0000-0002-5475-1788 \\ Donald Granados Gómez \\ dgranados@itcr.ac.cr \\ Instituto Tecnológico de Costa Rica \\ Costa Rica \\ https://orcid.org/0000-0003-1376-8898 \\ Nancy Sánchez Sulú \\ nancy.sulu@gmail.com \\ Universidad Autónoma del Carmen \\ https://orcid.org/0000-0003-2149-1374 \\ Yolanda Margarita Celi Vivanco \\ yolanda.celi@unl.edu.ec \\ Universidad Nacional de Loja \\ https://orcid.org/0000-0001-5969-5730
}

Recibido: 13 de mayo del 2019

Aprobado: 05 de junio del 2019

\section{RESUMEN}

El presente proyecto se desarrolló como parte de la red de contactos de docentes investigadores de Latinoamérica, quienes deciden desarrollar una propuesta conjunta sobre el uso del aula virtual en la Universidad Internacional del Ecuador, Tecnológico de Costa Rica y Universidad Autónoma del Carmen de México, describiendo la utilización del Moodle (Ecuador y México) y Tec digital (Costa Rica). La investigación fue de tipo descriptiva al analizar cómo es aprovechado el potencial que brindan los entornos virtuales de aprendizaje en la Universidad Internacional del Ecuador, la Universidad Autónoma del Carmen de México y el Tecnológico de Costa Rica, para lo cual se apoyó en un diseño 
no experimental. Se acepta la hipótesis nula que indica que las poblaciones investigadas aprovechan por igual las potencialidades de las TIC.

Descriptores: Aprendizaje en línea; Enseñanza asistida por ordenador; Informática educativa; Tecnología educacional.

\begin{abstract}
This project was developed as part of the network of contacts of Latin American research teachers, who decide to develop a joint proposal on the use of the virtual classroom at the International University of Ecuador, Technological University of Costa Rica and Universidad Autónoma del Carmen de México, describing the use of Moodle (Ecuador and Mexico) and Tec digital (Costa Rica). The research was descriptive in analyzing how the potential offered by virtual learning environments at the International University of Ecuador, the Autonomous University of Carmen of Mexico and the Technological University of Costa Rica is used, for which it relied on a design not experimental. The null hypothesis is accepted that indicates that the populations investigated take advantage of the potential of ICTs.
\end{abstract}

Descriptors: Electronic learning; Computer assisted instruction; Computer uses in education; Educational technology.

\title{
INTRODUCCIÓN
}

La propuesta detalla la utilización de la plataforma virtual en los países de Ecuador, Costa Rica y México, representados por las universidades participantes, con el objetivo de evaluar el uso de las plataformas virtuales en los periodos académicos 2017 - 2018, se origina como iniciativa de los docentes investigadores para analizar el trabajo en las aulas virtuales y cómo los estudiantes reaccionan al uso de esta herramienta tecnológica, según los diferentes escenarios. Esto plantea la siguiente pregunta de investigación: ¿Está siendo aprovechado el potencial que brindan los entornos virtuales de aprendizaje en la Universidad Internacional del Ecuador, la Universidad Autónoma del Carmen de México y el Tecnológico de Costa Rica? 
En este contexto, cabe destacar que la Universidad Internacional del Ecuador y la Universidad Autónoma del Carmen de México, usan la plataforma denominada Moodle; respecto a Ecuador, se empleó como apoyo a las materias de: Informática aplicada, investigación de mercados y métodos de investigación; en el caso de México, para los cursos presenciales de la academia de costos. En relación a Costa Rica, se utilizó el Tec Digital, para las materias de: Principios del diseño, procesos de manufactura y teoría del diseño.

Las herramientas tecnológicas, mencionadas, contribuyen al proceso de enseñanza aprendizajes facilitando la comunicación entre docente y estudiantes en cualquier lugar, y a cualquier hora, con un solo clic. Es así que se tuvo por objetivo general analizar cómo es aprovechado el potencial que brindan los entornos virtuales de aprendizaje en la Universidad Internacional del Ecuador, la Universidad Autónoma del Carmen de México y el Tecnológico de Costa Rica, lo cual permitió generar las siguientes hipótesis:

Hipótesis Nula0: Las poblaciones investigadas aprovechan por igual las potencialidades de las TIC

Hipótesis Alternativa1: Las poblaciones investigadas aprovechan diferencialmente las potencialidades de las TIC

\section{REFERENCIAL TEÓRICO}

\section{Entornos Virtuales de Aprendizaje}

Los entornos virtuales de enseñanza y aprendizaje ofrecen oportunidades ineludibles para el desarrollo de proyectos basados en metodologías de enseñanza-aprendizaje y trabajo cooperativo (Guitert \& Romeu, 2017). Son también considerados como "aulas sin paredes" siendo un espacio social virtual, cuyo mejor exponente actual es la Internet, así lo afirma (Belloch, citado en Fernández y Solano, 2009) no es presencial, sino representacional, no es proximal, sino distal, no es sincrónico, sino multicrónico, y no se basa en recintos espaciales con interior, frontera y exterior, sino que depende de redes 
electrónicas cuyos nodos de interacción pueden estar diseminados por diversos países. Así mismo Lattá Arias (2019), comenta que pueden ser herramientas utilizadas para el establecimiento de acciones que promuevan la integración de lo educativo con otras áreas como la productiva, siendo pertinente contar con esta modalidad de aprendizaje por cuanto permite no solo cumplir con actividades académicas, sino, para la vida.

Al respecto, los entornos de formación a distancia basados en las tecnologías de la información y comunicación señala (Salinas, 2004) que se apoyan en las decisiones relacionadas con el diseño de la enseñanza desde el punto de vista de la institución, docente y estudiante; así como en las decisiones que tienen que ver con la tecnología y selección de la herramienta de comunicación más adecuada.

Por otra parte (Ros, 2008) considera que los entornos virtuales de aprendizaje se tratan de una herramienta de E-learning, por cuanto posibilita el aprendizaje no presencial de los alumnos, aspecto este a considerar con muchos de los estudiantes que no pueden asistir a clases por su situación laboral o personal, lo que hace preciso contar con una herramienta que facilite la virtualidad; utilizando para este fin la red (internet e intranet) como tecnología de distribución de la información de dos formas según lo señala (Salmerón, 2010): como depósitos de información o como herramientas construidas para mejorar las oportunidades de interactividad propias de métodos de enseñanza activa que requieren especialmente de interacción para la co-construcción del conocimiento.

Existen algunas plataformas educativas como: Tiching, Edmodo, Blinklearning, RedAlumnos, Xtend, Educamos, estas comunidades se basan en una red social que comunica a padres, alumnos y profesores y permite a los docentes gestionar el contenido educativo, usan navegadores modernos unas van más allá con aplicaciones específicas para dispositivos móviles, y todas ellas soportan múltiples tipos y orígenes de contenidos educativos, según estudios realizados por Jm Tic (2015), sin embargo, Moodle por sus características, se 
ha constituido en la plataforma más utilizada a nivel mundial, contando con el registro de: 94,471 sitios, 231 países, 126,555,549 usuarios, etcétera, datos evidenciados en las estadísticas del sitio Web oficial Moodle (Moodle, 2018).

La variedad de plataformas tecnológicas que integran un entorno virtual de aprendizaje es numerosa, sin embargo según (Vega Gil, 2010) se pueden clasificar en tres grupos: desarrollos propios, plataformas comerciales 0 plataformas de software libre. En el presente caso, relacionado a Costa Rica, el Moodle corresponde a una plataforma comercial, el Tec Digital es una mezcla entre una plataforma de software libre y una de desarrollo propio, por cuanto la universidad ha desarrollado software que se ha integrado en la de software libre.

Por otro lado, Moodle es un sistema para el manejo del aprendizaje en línea gratuito, que les permite a los educadores la creación de sus propios sitios web privados, llenos de cursos dinámicos que extienden el aprendizaje, en cualquier momento, en cualquier sitio. Según la información encontrada el su sitio web (Moodle, 2018), el núcleo de Moodle, extremadamente personalizable, viene con muchas características estándar. A continuación, las características principales del núcleo de Moodle. Existen 94,48 sitios actualmente activos, se han registrado de 231 países, con 126,670,699 usuarios, llevándose hasta la actualidad 15,036,031 información que se evidencia en el sitio web oficial de Moodle (2018).

Top 10 de los sitios registrados en 231 países

\section{Cuadro 1}

\begin{tabular}{llc} 
Nro. & País & Inscripciones \\
\hline 1 & Estados Unidos & 10,034 \\
2 & España & 8,093 \\
3 & México & 5,276 \\
4 & Brasil & 5,076 \\
5 & Reino Unido & 3,502 \\
6 & Alemania & 3,026 \\
7 & Italia & 2,902 \\
8 & Colombia & 2,698 \\
9 & Federación Rusa & 2,529
\end{tabular}


Fuente: Sitio web oficial de Moodle, 2018

En este entorno, la importancia atribuida a la asistencia educativa, al principio de ajuste del apoyo para la comprensión de los procesos de enseñanza y aprendizaje en entornos virtuales aconseja ir más allá de un modelo de análisis y explicación de esos procesos basado únicamente en la interacción entre aprendiz, contenido, sustituirlo por un modelo más amplio, basado en la relación entre tres elementos: la actividad mental constructiva del alumno que aprende, la ayuda sostenida y continuada del que enseña, el contenido que es objeto de enseñanza y aprendizaje, sin embargo es necesario tener en consideración lo previsto por Urueta Vélez (2019), al indicar la necesidad que los docentes se encuentren formados para asumir estilos educativos basados en lo significativo como el aprendizaje generado desde las TIC. Así mismo Barrientos Oradini, Araya Castillo, Herrera Ciudad \& Muñoz Morales (2019), destacan la importancia de motivar al docente al uso adecuado de las TIC, por cuanto es este quien procederá a transmitir el empleo de las mismas a los estudiantes, con lo cual se deben evitar improvisaciones que puedan afectar el rendimiento académico de los estudiantes.

\section{METODOLOGÍA DE LA INVESTIGACIÓN}

La investigación fue de tipo descriptiva al analizar cómo es aprovechado el potencial que brindan los entornos virtuales de aprendizaje en la Universidad Internacional del Ecuador, la Universidad Autónoma del Carmen de México y el Tecnológico de Costa Rica, para lo cual se apoyó en un diseño no experimental, (Hernández Sampieri, Fernández Collado \& Baptista 2014), plantean que esta tipología permite realizar análisis comparativos entre un objeto de estudio con la finalidad de describirlo tal como ocurre en la realidad. Se trabajó durante los periodos académicos 2017 - 2018, con la participación de 50 estudiantes de Ecuador, en las materias de: informática aplicada, investigación de mercados y métodos de investigación; 53 educandos de costa 
rica, en las asignaturas de: principios del diseño, procesos de manufactura 3 y teoría del diseño 3; y 55 de México, en la materia de costos de manufactura para las diferentes carreras de la facultad de administración.

Se llevó a cabo la investigación en las instalaciones de la Universidad Internacional del Ecuador, extensión Loja, Tecnológico de Costa Rica y Universidad Autónoma de México utilizándose la plataforma virtual Moodle, para las Universidades participantes de Ecuador y México, y TEC Digital en el caso de Costa Rica, en vista que las instituciones mencionadas trabajan con ese tipo de plataforma virtual. Estadísticamente se empleó tablas de contingencia o tablas cruzadas y el análisis de varianza (ANOVA) de un factor, esto permitió establecer las siguientes hipótesis:

Hipótesis Nula0: Las poblaciones investigadas aprovechan por igual las potencialidades de las TIC

Hipótesis Alternativa1: Las poblaciones investigadas aprovechan diferencialmente las potencialidades de las TIC

\section{RESULTADOS}

En el presente epígrafe se realiza la presentación de las tablas de contingencia o tablas cruzadas y el análisis de varianza (ANOVA) de un factor, obteniendo los siguientes resultados a continuación descritos, pero, antes de aquello es importante señalar que en todas las preguntas se repite los valores del resumen de procesamiento de casos, por tal motivo, se han considerado exclusivamente para la pregunta número uno.

Tabla cruzada ¿Con qué frecuencia hace uso de la plataforma virtual?País

\section{Cuadro 2}

Fuente: Entrevistados 


\begin{tabular}{llrrrr} 
& & \multicolumn{2}{c}{$\begin{array}{c}\text { País } \\
\text { Costa Rica }\end{array}$} & México & \multicolumn{2}{c}{ Total } \\
\hline ¿Con qué frecuencia & Una vez al día & 11 & 4 & 19 & 34 \\
hace uso de la plataforma & & 20 & 36 & 12 & 68 \\
virtual? & $\begin{array}{l}\text { Varias veces al día } \\
\text { Hasta cinco veces }\end{array}$ & 12 & 12 & 17 & 41 \\
& $\begin{array}{l}\text { Por semana } \\
\text { Una vez por }\end{array}$ & 2 & 1 & 5 & 8 \\
& $\begin{array}{l}\text { Semana } \\
\text { Los fines de }\end{array}$ & 5 & 0 & 2 & 7 \\
Total & semana & 50 & 53 & 55 & 158 \\
\hline
\end{tabular}

Según la tabla presentada se evidencia que en los países participantes, sus estudiantes utilizan en un porcentaje considerable la plataforma virtual, varias veces al día y otros hasta cinco veces por semana. Es notorio en el caso de Costa Rica que más de la mitad de los encuestados revisan la plataforma varias veces al día, mientras en México lo hace aproximadamente la tercera parte. En el caso de México se tiene que aproximadamente la tercera parte de los estudiantes la revisan hasta cinco veces por semana, mientras que en Ecuador y Costa Rica rondan la cuarta parte de los entrevistados. Es claro al revisar los totales que la amplia mayoría de los estudiantes revisan al menos cinco veces a la semana sus Entornos Virtuales de Aprendizaje, alcanzando un pico máximo en la descripción "Varias veces al día".

¿Con qué frecuencia hace uso de la plataforma virtual?

\section{Cuadro 3}

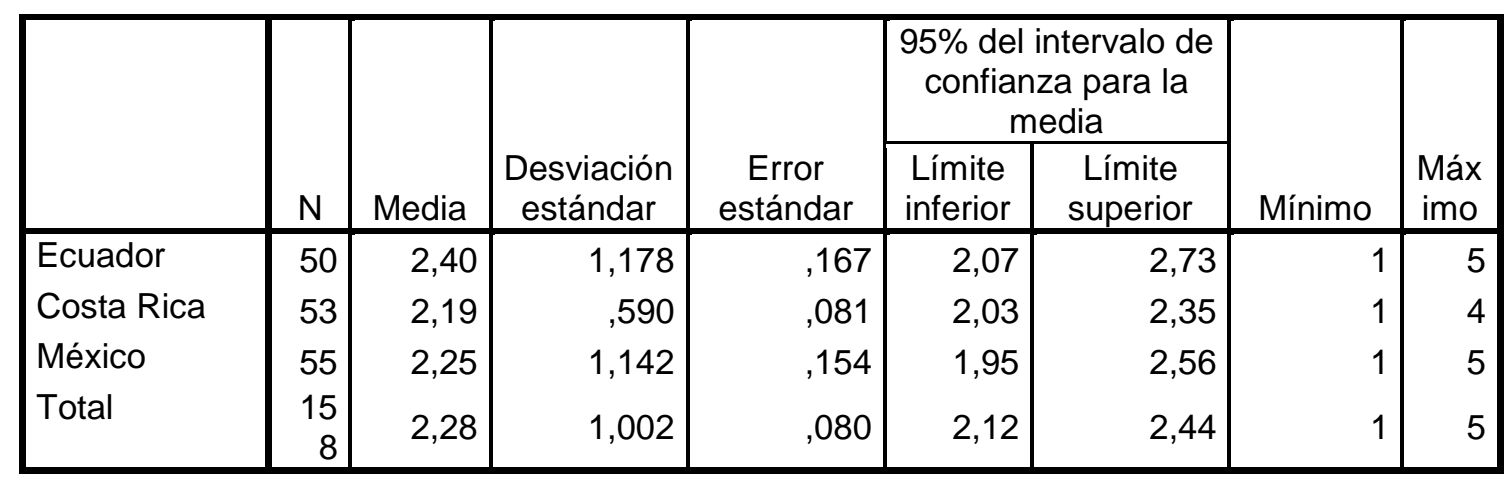

Fuente: Encuestados 
Se evidencia que la media de la frecuencia de uso de la plataforma virtual es diferente según cada país, sea Ecuador, Costa Rica o México. Las desviaciones estándar son moderadas, pues tanto los estudiantes de género masculino y femenino de los países participantes, usan con frecuencia la plataforma virtual.

¿Con qué frecuencia hace uso de la plataforma virtual?

\section{Cuadro 4}

\begin{tabular}{|c|c|c|c|c|c|}
\hline & $\begin{array}{l}\text { Suma de } \\
\text { cuadrados }\end{array}$ & gl & Media cuadrática & $\mathrm{F}$ & Siq. \\
\hline Entre grupos & 1,197 & 2 & ,599 & ,593 & ,554 \\
\hline Dentro de grupos & 156,550 & 155 & 1,010 & & \\
\hline Total & 157,747 & 157 & & & \\
\hline
\end{tabular}

Fuente: Encuestados

En la tabla del ANOVA se observa la suma de cuadrados entre grupos, su valor 1,197 y los grados de libertad que le corresponden. Dividiendo esta suma de cuadrados por los grados de libertad 2, se obtiene la media cuadrática 0,599 que es el numerador de la fórmula del estadístico F. La suma de cuadrados dentro de los grupos, su valor 156,550 y los grados de libertad que le corresponden. Dividiendo esta suma de cuadrados por los grados de libertad 155, se obtiene la otra media cuadrática 1,010 que es el denominador de la fórmula del estadístico $F$.

La significación de F es mayor que 0,05, por tanto, se acepta la hipótesis nula, concluyendo que la media de la frecuencia de uso de la plataforma virtual es similar entre los países de Ecuador, Costa Rica y México.

¿Cuál es el motivo por el cual usted hace uso indispensable de la plataforma virtual para el desarrollo de su preparación académica?*País

\section{Cuadro 5}




\begin{tabular}{|c|c|c|c|c|c|}
\hline & & Ecuador & Costa Rica & México & \\
\hline $\begin{array}{l}\text { ¿Cuál es el motivo } \\
\text { por el cual usted }\end{array}$ & $\begin{array}{l}\text { Consulta en base de } \\
\text { datos }\end{array}$ & 2 & 13 & 20 & 35 \\
\hline $\begin{array}{l}\text { hace uso } \\
\text { indispensable de la }\end{array}$ & $\begin{array}{l}\text { Observar temarios } \\
\text { vistos en clase }\end{array}$ & 10 & 29 & 12 & 51 \\
\hline $\begin{array}{l}\text { plataforma virtual } \\
\text { para el desarrollo de }\end{array}$ & $\begin{array}{l}\text { Conocer los temas y } \\
\text { tareas a futuro }\end{array}$ & 18 & 10 & 15 & 43 \\
\hline $\begin{array}{l}\text { su preparación } \\
\text { académica? }\end{array}$ & $\begin{array}{l}\text { Entregar informes y } \\
\text { tareas }\end{array}$ & 20 & 1 & 8 & 29 \\
\hline Total & & 50 & 53 & 55 & 158 \\
\hline
\end{tabular}

\section{Fuente: Encuestados}

En la tabla se analiza que los estudiantes en un alto porcentaje, usan la plataforma virtual para el desarrollo de su preparación académica; en Ecuador para entregar informes y tareas, Costa Rica para Observar temarios vistos en clase y México para consultar en base de datos. Puede advertirse en el total de los datos que la mayor parte de los estudiantes de las tres universidades hacen mayor uso de las plataformas para ver los temarios vistos en clase. Esto habla de la revisión posterior de materia teórica o de temas de clases pasadas que probablemente sean empleados para el estudio o la elaboración de tareas y proyectos. Seguidamente está el conocer los temas y tareas a futuro, lo cual podría estar referido a la necesidad de planificar el tiempo académico para hacer un uso acertado del mismo.

En un tercer lugar está la consulta en bases de datos, probablemente relacionado con el desarrollo de actividades propias del curso. Cabe mencionar que en este rubro la universidad en Ecuador obtiene un valor considerado bajo si se le compara con los resultados de México y Costa Rica. Resulta importante hacer un estudio del caso específico para conocer las causas que generan ese resultado.

¿Cuál es el motivo por el cual usted hace uso indispensable de la plataforma virtual para el desarrollo de su preparación académica?

\section{Cuadro 6}

N Media $\begin{gathered}\text { Desviación } \\ \text { estándar }\end{gathered} \quad \begin{gathered}\text { Error } \\ \text { estándar }\end{gathered} \begin{gathered}\text { 95\% del intervalo de } \\ \text { confianza para la } \\ \text { media }\end{gathered}$ Mínimo Máximo




\begin{tabular}{lrrrrrrrr} 
& & \multicolumn{8}{c}{$\begin{array}{c}\text { Límite } \\
\text { inferior }\end{array}$} & $\begin{array}{c}\text { Límite } \\
\text { superior }\end{array}$ \\
\hline Ecuador & 50 & 3,12 &, 872 &, 123 & 2,87 & 3,37 & 1 & 4 \\
Costa Rica & 53 & 1,98 &, 720 &, 099 & 1,78 & 2,18 & 1 & 4 \\
México & 55 & 2,20 & 1,095 &, 148 & 1,90 & 2,50 & 1 & 4 \\
Total & 15 & 2,42 & 1,030 &, 082 & 2,26 & 2,58 & 1 & 4 \\
& 8 & & & &
\end{tabular}

Fuente: Encuestados

Los descriptivos, muestra que la media de uso de la plataforma virtual para el desarrollo de la preparación académica de los estudiantes, es diferente si se eligen desde los distintos países. Las desviaciones estándar son moderadas, no existe mayor diferencia entre las variables, pero al margen de aquello, se puede observar que los estudiantes de Ecuador utilizan en mayor proporción el aula virtual para entregar informes y tareas.

¿Cuál es el motivo por el cual usted hace uso indispensable de la plataforma virtual para el desarrollo de su preparación académica?

\section{Cuadro 7}

\begin{tabular}{lrrrrr} 
& \multicolumn{1}{c}{$\begin{array}{c}\text { Suma de } \\
\text { cuadrados }\end{array}$} & \multicolumn{2}{c}{$\begin{array}{c}\text { Media } \\
\text { cuadrática }\end{array}$} & \multicolumn{1}{c}{ F } & \multicolumn{1}{c}{ Sig. } \\
\hline Entre grupos & 37,369 & 2 & 18,685 & 22,440 &, 000 \\
Dentro de grupos & 129,061 & 155 &, 833 & & \\
Total & 166,430 & 157 & & & \\
\hline
\end{tabular}

Fuente: Encuestados

Obteniendo el siguiente resultado, lo que interesa es la significación de $\mathrm{F}$ que es menor de 0,05 ya que toma el valor de 0,00, por lo que se rechaza la hipótesis nula y se acepta la hipótesis alternativa, por tanto, se concluye que el motivo indispensable del uso de la plataforma virtual para la preparación académica es diferente considerando los motivos para su uso, entre y dentro de los países participantes. 
¿Cuál es el motivo por el cual usted hace uso indispensable de la plataforma virtual para el desarrollo de su preparación académica?

\section{Cuadro 8}

\begin{tabular}{|c|c|c|c|c|c|c|}
\hline \multirow[b]{2}{*}{ (I) País } & \multirow[b]{2}{*}{ (J) País } & \multirow[b]{2}{*}{$\begin{array}{l}\text { Diferencia de } \\
\text { medias (I-J) }\end{array}$} & \multirow[b]{2}{*}{$\begin{array}{l}\text { Error } \\
\text { estándar }\end{array}$} & \multirow[b]{2}{*}{ Sig. } & \multicolumn{2}{|c|}{$\begin{array}{l}\text { Intervalo de confianza al } \\
95 \%\end{array}$} \\
\hline & & & & & $\begin{array}{l}\text { Límite } \\
\text { inferior }\end{array}$ & $\begin{array}{l}\text { Límite } \\
\text { superior }\end{array}$ \\
\hline Ecuador & $\begin{array}{l}\text { Costa } \\
\text { Rica }\end{array}$ & $1,139^{*}$ & 158 & ,000 & ,76 & 1,52 \\
\hline $\begin{array}{l}\text { Costa } \\
\text { Rica } \\
\text { México }\end{array}$ & $\begin{array}{l}\text { México } \\
\text { Ecuador } \\
\text { México } \\
\text { Ecuador }\end{array}$ & $\begin{array}{r}, 920^{*} \\
-1,139^{*} \\
-, 219 \\
-, 920^{*}\end{array}$ & $\begin{array}{l}, 192 \\
, 158 \\
, 178 \\
, 192\end{array}$ & $\begin{array}{l}, 000 \\
, 000 \\
, 438 \\
, 000\end{array}$ & $\begin{array}{r}, 46 \\
-1,52 \\
-, 64 \\
-1,38\end{array}$ & $\begin{array}{r}1,38 \\
-, 76 \\
, 20 \\
-, 46\end{array}$ \\
\hline & $\begin{array}{l}\text { Costa } \\
\text { Rica }\end{array}$ & 219 & 178 & ,438 &,- 20 & 64 \\
\hline
\end{tabular}

*. La diferencia de medias es significativa en el nivel 0.05 .

Fuente: Encuestados

La prueba compara entre grupos y se lee de la siguiente manera: El grupo 1, si se le compara con el grupo 2, la significación es 0,00 valores menores a 0,050 por lo que se rechaza la hipótesis nula. Ello implica que las medias del motivo por el cual hacen uso indispensable de la plataforma virtual para el desarrollo de la preparación académica, es diferente. De igual manera sucede si se compara el grupo uno con el tres, cuya significación es la misma que en al anterior caso. 
¿Qué actividad promueve con mayor interés los docentes a través de la plataforma virtual?*País

\section{Cuadro 9}

\begin{tabular}{|c|c|c|c|c|c|}
\hline & & Ecuador & $\begin{array}{c}\text { País } \\
\text { Costa Rica }\end{array}$ & México & Total \\
\hline \multirow{5}{*}{$\begin{array}{l}\text { ¿Qué actividad } \\
\text { promueve con } \\
\text { mayor interés los } \\
\text { docentes a través de } \\
\text { la plataforma virtual? }\end{array}$} & Foro & 1 & 0 & 28 & 29 \\
\hline & $\begin{array}{l}\text { Subir archivos de } \\
\text { tareas en clase y } \\
\text { extraclase } \\
\text { Subir material }\end{array}$ & 41 & 31 & 9 & 81 \\
\hline & $\begin{array}{l}\text { didáctico de las } \\
\text { clases }\end{array}$ & 4 & 22 & 11 & 37 \\
\hline & Tareas en línea & 3 & 0 & 7 & 10 \\
\hline & En blanco & 1 & 0 & 0 & \\
\hline Total & & 50 & 53 & 55 & 158 \\
\hline
\end{tabular}

Fuente: Entrevistados

Se observa en la tabla en un alto porcentaje, que la actividad promovida con mayor interés a través del aula virtual por los docentes, para los estudiantes de Ecuador y Costa Rica, es la de subir archivos y tareas en clase y extraclase; mientras que, para México es la participación en foros. Es notorio que tanto en Ecuador como en Costa Rica se obtienen un valor bajo o nulo en los foros, eso parece indicar una subutilización de la utilidad del Entorno Virtual de Aprendizaje. Mientras, por su parte en México el valor más bajo se encuentra en la actividad de realizar tareas en línea. 
¿Qué actividad promueve con mayor interés los docentes a través de la plataforma virtual?

\section{Cuadro 10}

\begin{tabular}{|c|c|c|c|c|c|c|c|c|}
\hline & \multirow[b]{2}{*}{$\mathrm{N}$} & \multirow[b]{2}{*}{ Media } & \multicolumn{6}{|c|}{$\begin{array}{c}95 \% \text { del intervalo de } \\
\text { confianza para la } \\
\text { media }\end{array}$} \\
\hline & & & $\begin{array}{l}\text { Desviación } \\
\text { estándar }\end{array}$ & $\begin{array}{l}\text { Error } \\
\text { estándar }\end{array}$ & $\begin{array}{l}\text { Límite } \\
\text { inferior }\end{array}$ & $\begin{array}{l}\text { Límite } \\
\text { superior }\end{array}$ & Mínimo & Máximo \\
\hline Ecuador & 50 & 4,12 & 13,703 & 1,938 & ,23 & 8,01 & 1 & 99 \\
\hline $\begin{array}{l}\text { Costa } \\
\text { Rica }\end{array}$ & 53 & 2,42 & ,497 & ,068 & 2,28 & 2,55 & 2 & 3 \\
\hline México & 55 & 1,95 & 1,113 & ,150 & 1,64 & 2,25 & 1 & 4 \\
\hline Total & 158 & 2,79 & 7,744 & ,616 & 1,57 & 4,01 & 1 & 99 \\
\hline
\end{tabular}

Fuente: Entrevistados

La media de la actividad promovida con mayor interés por los docentes a través de la plataforma virtual, es diferente si se eligen desde los distintos países. Las desviaciones estándar en Ecuador son muy altas, no existiendo diferencia entre las variables, pero, al margen de aquello, se puede destacar que a los estudiantes de Ecuador son quienes utilizan en mayor proporción el aula virtual para Subir archivos de tareas en clase y extraclase, seguidos por los estudiantes de Costa Rica.

¿Qué actividad promueve con mayor interés los docentes a través de la plataforma virtual?

\section{Cuadro 11}

\begin{tabular}{llllll}
\hline & Suma & de & & Media & \\
& cuadrados & gl & cuadrática & $\mathrm{F}$ & Sig. \\
Entre grupos & 135,123 & 2 & 67,562 & 1,128 &, 326 \\
\hline
\end{tabular}




\begin{tabular}{llll}
\hline Dentro de grupos & 9280,984 & 155 & 59,877 \\
Total & 9416,108 & 157 & \\
\hline
\end{tabular}

Fuente: Entrevistados

En la tabla del ANOVA se observa la suma de cuadrados entre grupos, su valor 135,123 y los grados de libertad que le corresponden. Dividiendo esta suma de cuadrados por los grados de libertad 2, se obtiene la media cuadrática 67,562 que es el numerador de la fórmula del estadístico F. La suma de cuadrados dentro de los grupos, su valor 9280,984 y los grados de libertad que le corresponden. Dividiendo esta suma de cuadrados por los grados de libertad 155, se obtiene la otra media cuadrática 59,877 que es el denominador de la fórmula del estadístico $\mathrm{F}$.

La significación de $\mathrm{F}$ es 0,326 mayor que 0,05, por tanto, se acepta la hipótesis nula, concluyendo que la media de la actividad que promueven los docentes con mayor interés, a través de la plataforma virtual, es similar entre los países de Ecuador, Costa Rica y México.

¿Cómo calificaría usted esta nueva herramienta virtual utilizada en la Universidad?*País

\section{Cuadro 12}

\begin{tabular}{|c|c|c|c|c|c|}
\hline & & Ecuador & $\begin{array}{l}\text { País } \\
\text { Costa } \\
\text { Rica }\end{array}$ & México & Total \\
\hline ¿Cómo calificaría & $\begin{array}{l}\text { Muy } \\
\text { deficiente }\end{array}$ & 3 & 0 & 6 & 9 \\
\hline herramienta virtual & Deficiente & 4 & 3 & 7 & 14 \\
\hline utilizada en la & Regular & 19 & 21 & 11 & 51 \\
\hline Universidad? & $\begin{array}{l}\text { Buena } \\
\text { Excelente }\end{array}$ & $\begin{array}{r}18 \\
6\end{array}$ & $\begin{array}{r}27 \\
2\end{array}$ & $\begin{array}{l}19 \\
12\end{array}$ & $\begin{array}{l}64 \\
20\end{array}$ \\
\hline Total & & 50 & 53 & 55 & 158 \\
\hline
\end{tabular}

Fuente: Entrevistados

Se observa en la tabla que un mayor porcentaje de participantes de Ecuador, Costa Rica y México, califican a la herramienta virtual, como regular y buena; 
analizándose un mínimo porcentaje que la catalogan como deficiente y otros eficientes.

¿Cómo calificaría usted esta nueva herramienta virtual utilizada en la Universidad?

\section{Cuadro 13}

\begin{tabular}{|c|c|c|c|c|c|c|c|c|}
\hline & \multirow[b]{2}{*}{$N$} & \multirow[b]{2}{*}{ Media } & \multirow[b]{2}{*}{$\begin{array}{l}\text { Desviación } \\
\text { estándar }\end{array}$} & \multirow[b]{2}{*}{$\begin{array}{c}\text { Error } \\
\text { estándar }\end{array}$} & \multicolumn{2}{|c|}{$\begin{array}{c}95 \% \text { del intervalo de } \\
\text { confianza para la media }\end{array}$} & \multirow[b]{2}{*}{ Mínimo } & \multirow[b]{2}{*}{ Máximo } \\
\hline & & & & & $\begin{array}{l}\text { Límite } \\
\text { inferior }\end{array}$ & $\begin{array}{l}\text { Límite } \\
\text { superior }\end{array}$ & & \\
\hline Ecuador & 50 & 3,40 & 1,010 & ,143 & 3,11 & 3,69 & 1 & 5 \\
\hline $\begin{array}{l}\text { Costa } \\
\text { Rica }\end{array}$ & 53 & 3,53 & 668 & ,092 & 3,34 & 3,71 & 2 & 5 \\
\hline México & 55 & 3,44 & 1,273 & ,172 & 3,09 & 3,78 & 1 & 5 \\
\hline Total & 158 & 3,46 & 1,013 & ,081 & 3,30 & 3,61 & 1 & 5 \\
\hline
\end{tabular}

Fuente: Entrevistados

En la presente tabla, los descriptivos, señalan que la media de la calificación de la herramienta virtual utilizada en la Universidad es diferente si se eligen desde los distintos países. Las desviaciones estándar en Ecuador son moderadas, no existiendo diferencia entre las variables, pero, al margen de aquello, se puede destacar que los estudiantes de Ecuador son quienes utilizan en mayor proporción el aula virtual para Subir archivos de tareas en clase y extraclase, seguidos por los estudiantes de Costa Rica.

¿Cómo calificaría usted esta nueva herramienta virtual utilizada en la Universidad?*País

\section{Cuadro 14} Sig. 


\begin{tabular}{lrrrrr}
\hline Entre grupos &, 455 & 2 &, 228 &, 219 &, 803 \\
Dentro de grupos & 160,735 & 155 & 1,037 & & \\
Total & 161,190 & 157 & & & \\
\hline
\end{tabular}

Fuente: Entrevistados

En la tabla del ANOVA se observa la suma de cuadrados entre grupos, su valor 0,455 y los grados de libertad que le corresponden. Dividiendo esta suma de cuadrados por los grados de libertad 2, se obtiene la media cuadrática 0,228 que es el numerador de la fórmula del estadístico $\mathrm{F}$.

La suma de cuadrados dentro de los grupos, su valor 160,735 y los grados de libertad que le corresponden. Dividiendo esta suma de cuadrados por los grados de libertad 155, se obtiene la otra media cuadrática 1,037 que es el denominador de la fórmula del estadístico $\mathrm{F}$.

La significación de $\mathrm{F}$ es 0,803 mayor que 0,05 , por tanto, se acepta la hipótesis nula, concluyendo que la media de la actividad que promueven los docentes con mayor interés, a través de la plataforma virtual, es similar entre los países de Ecuador, Costa Rica y México.

Tabla cruzada Clasifique según su criterio si la plataforma virtual es una buena herramienta de estudio a la hora de presentar un trabajo?*País

\section{Cuadro 15}

\begin{tabular}{|c|c|c|c|c|c|}
\hline & & Fourdor & $\begin{array}{l}\text { País } \\
\text { Costa }\end{array}$ & Máxicn & Total \\
\hline ¿Clasif & Fxcelente & $\frac{\text { ador }}{8}$ & $\frac{a}{10}$ & $\begin{array}{r}\text { Mlexico } \\
20\end{array}$ & $\begin{array}{r}\text { Total } \\
38\end{array}$ \\
\hline criter & Bue & 27 & 30 & 15 & 72 \\
\hline plataforma virtual es & Regular & 10 & 12 & 16 & 38 \\
\hline una buena & Mala & 3 & 1 & 3 & $r$ \\
\hline $\begin{array}{l}\text { herramienta de } \\
\text { estudio a la hora de }\end{array}$ & $\begin{array}{l}\text { Innecesari } \\
\mathrm{a}\end{array}$ & 1 & 0 & 1 & \\
\hline $\begin{array}{l}\text { presentar un trabajo? } \\
\text { Total }\end{array}$ & En blanco & $\begin{array}{r}1 \\
50\end{array}$ & $\begin{array}{r}0 \\
53\end{array}$ & $\begin{array}{r}0 \\
55\end{array}$ & $\begin{array}{r}1 \\
158\end{array}$ \\
\hline
\end{tabular}

Fuente: Entrevistados 
En la tabla se evidencia que un mayor porcentaje de participantes de Ecuador y Costa Rica, consideran que la plataforma virtual es buena herramienta de estudio a la hora de presentar un trabajo; mientras que los estudiantes de México, la consideran como excelente. Se observa en cambio que un mínimo porcentaje entre los tres países que la catalogan como mala herramienta.

¿Clasifique según su criterio si la plataforma virtual es una buena herramienta de estudio a la hora de presentar un trabajo?

\section{Cuadro 16}

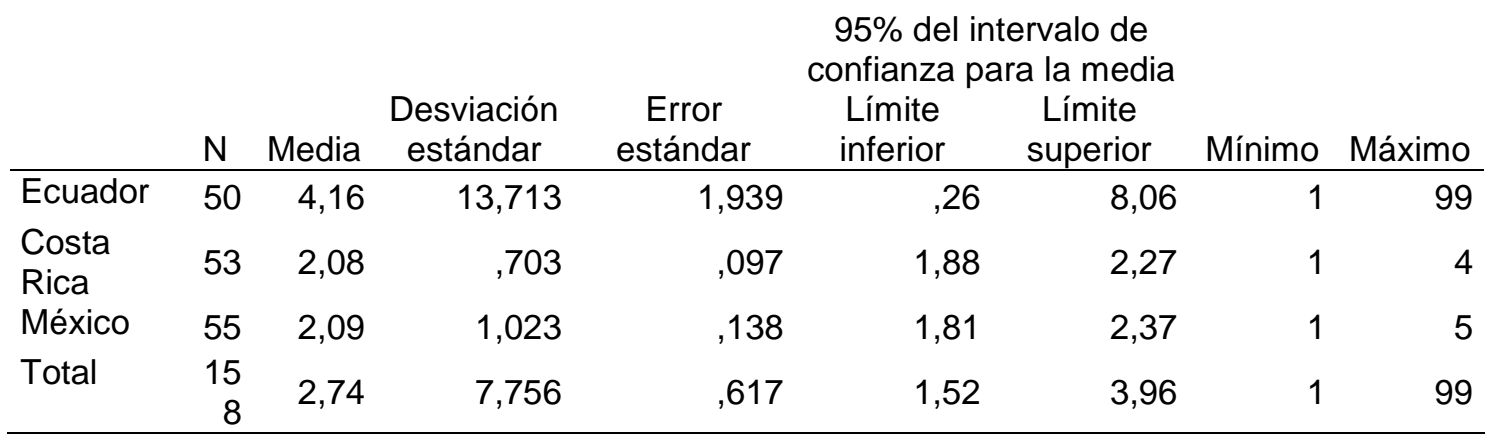

Fuente: Entrevistados

En los descriptivos, se analiza que la media de la calificación de la herramienta virtual utilizada en la Universidad, es diferente si se eligen desde los distintos países. Las desviaciones estándar en Ecuador son altas, la información indica que los estudiantes de Ecuador son quienes más han considerado como buena la utilización de la herramienta de estudio a la hora de presentar un trabajo.

¿Clasifique según su criterio si la plataforma virtual es una buena herramienta de estudio a la hora de presentar un trabajo?

Cuadro 17

\begin{tabular}{|c|c|c|c|c|c|}
\hline & $\begin{array}{c}\text { Suma de } \\
\text { cuadrados }\end{array}$ & gl & $\begin{array}{c}\text { Media } \\
\text { cuadrática }\end{array}$ & $\mathrm{F}$ & Sig. \\
\hline Entr & 147,397 & 2 & 73,699 & 1,229 & ,296 \\
\hline grupos & 9296,964 & 155 & 59,980 & & \\
\hline Total & 9444,361 & 157 & & & \\
\hline
\end{tabular}

Fuente: Entrevistados 
En la tabla del ANOVA se observa la suma de cuadrados entre grupos, su valor 147,397 y los grados de libertad que le corresponden. Dividiendo esta suma de cuadrados por los grados de libertad 2, se obtiene la media cuadrática 73,699 que es el numerador de la fórmula del estadístico $\mathrm{F}$.

La suma de cuadrados dentro de los grupos, su valor 9296,964 y los grados de libertad que le corresponden. Dividiendo esta suma de cuadrados por los grados de libertad 155, se obtiene la otra media cuadrática 59,980 que es el denominador de la fórmula del estadístico $\mathrm{F}$.

La significación de $F$ es 0,296 mayor que 0,05, por tanto, se acepta la hipótesis nula, concluyendo que la media de la plataforma virtual es una buena herramienta de estudio a la hora de presentar un trabajo, criterio similar entre los países de Ecuador, Costa Rica y México.

Las plataformas virtuales se constituyen en herramientas de enseñanza aprendizaje para el fortalecimiento del proceso educativo universitario, información corroborada en base a los resultados obtenidos en la investigación, la cual expresa que los estudiantes de las instituciones de educación superior, consideran que el aula virtual es una buena herramienta de estudio, obteniendo una significación de 0,269 en el análisis del ANOVA de un factor, valor mayor a 0,050 , por lo tanto se acepta la hipótesis nula.

En la propuesta es importante destacar la participación de 158 estudiantes distribuidos entre la Universidad Internacional del Ecuador, Tecnológico de Costa Rica y Universidad Autónoma del Carmen de México, se observa 0\% de valores perdidos, según el resumen de procesamiento de datos, facilitado por el programa estadístico SPSS.

El trabajo se constituye en un aporte para la educación universitaria y quienes deseen seguir incursionando en este tema, con proyección a utilizar las redes de contactos para generar investigación entre países, de esa manera se conocerán las diversas realidades suscitadas en cuanto al uso de las plataformas virtuales en el proceso educativo universitario. 
Las plataformas virtuales son empleadas por los estudiantes, de preferencia varias veces al día y en algunos casos hasta cinco veces por semana, siendo la media de frecuencia diferente según el país analizado, por ejemplo, en Ecuador se observa una media de 2,40, seguida de Costa Rica con 2,19 y México 2,25. Esto parece indicar que los docentes emplean las plataformas de manera tal que generan en el estudiante la necesidad de visitarlas para la satisfacción de alguna necesidad relacionada con el aprendizaje. Por tanto, evidencia que la plataforma constituye parte de la estrategia didáctica y que una gran cantidad de estudiantes deben visitarla para obtener un beneficio en su uso.

Por otra parte, cabe destacar que los estudiantes de Ecuador en una gran mayoría, utilizan el aula virtual para entregar informes y tareas, mientras que los alumnos de Costa Rica se enfocan más a observar temarios vistos en clase, no así los de México la emplean para los foros. Ante tal resultado, se deben desarrollar estrategias de trabajo con la finalidad se use de manera apropiada el aula virtual, aprovechando sus beneficios como son consulta de datos, conocer los temas y tareas a futuro, a más de los indicados.

Lo expresado, también se sustenta en la información que arroja el SPSS, evidenciándose que la actividad que promueven con mayor interés los maestros, a través del aula virtual en Ecuador y Costa Rica es la subir archivos de tareas en clase y extraclase; mientras que, para México, su empleo en los foros. El uso en Ecuador parece estar referido al cumplimento en entrega de medios de evaluación, mientras que en Costa Rica puede interpretarse que se da una revisión posterior de materia teórica o de temas de clases pasadas que probablemente sean empleados para el estudio o la elaboración de tareas y proyectos.

Mientras tanto en México se emplea mayoritariamente los foros, lo cual puede indicar el desarrollo de una actividad complementaria o de evaluación que permite la construcción de conocimiento mediante la interacción de estudiantes. En cuanto a la opinión externada por los estudiantes respecto de 
la calificación de la herramienta la evalúan como regular y buena. Es importante hacer notar en el total de los datos que la impresión positiva, formada por los descriptores "Buena" y "Excelente" son contundentemente altos si se comparan con la impresión negativa, formada por los descriptores "Deficiente" y "Muy deficiente". Sin embargo, no se puede pasar por alto que existe también un número elevado en la posición de indiferencia, dado por el descriptor "Regular", cercano a la tercera parte de las personas encuestadas. $Y$ que estos resultados reflejan un resumen de la impresión total de los estudiantes en los tres países, ese resultado no es trasladable a cada país en particular.

Al preguntar si consideran que la plataforma es buena se concluye que un mayor porcentaje de participantes de Ecuador y Costa Rica, consideran que la plataforma virtual es buena mientras que los estudiantes de México, excelente. Puede notarse que, en términos de la impresión positiva, formada por los descriptores "Buena" y "Excelente" se tiene un alto porcentaje de la muestra, arriba del $70 \%$, mientras que la impresión negativa dada por los descriptores "Mala" e "Innecesaria" tienen un valor considerado bajo, apenas superior al 5\%. En la posición de la indiferencia, dada por el descriptor "Regular" se tiene aproximadamente la cuarta parte de los encuestados. Es probable que los estudiantes consideren la plataforma como un mínimo esperable en el proceso y por ese motivo no perciben les otorgue valor al presentar un trabajo.

Con esta información se llega a la conclusión que la plataforma está siendo subutilizada, obviamente, se la está empleando como una herramienta aprendizaje, indiscutiblemente es un aporte para la educación universitaria, sin embargo, se la podría utilizar considerando todos los beneficios que la misma ofrece. Por tanto, se considera debe promoverse el uso del aula virtual en todos sus componentes, esto obviamente, demandará más tiempo y dedicación de los docentes y estudiantes y desde esta óptica, ya se está planteando una nueva hipótesis, así como la posibilidad de nuevos experimentos, para una futura investigación. 


\section{CONCLUSIONES}

A partir de la información anterior puede concluirse lo siguiente:

Los estudiantes utilizan frecuentemente las plataformas en cada una de las universidades.

Las actividades que más frecuentemente emplean los estudiantes son diferentes para cada universidad, en Ecuador para entregar informes y tareas, en Costa Rica para observar temarios de clase y en México para la realización de Foros.

Los estudiantes de las tres universidades parecen tener una impresión positiva al asignar una calificación a la herramienta virtual de su universidad, declarándola como "Buena" y "Excelente"

La percepción general que tienen los estudiantes acerca de la plataforma como herramienta de estudio es positiva, teniendo un mayor valor en los descriptores "Bueno" y "Excelente"

Tanto la calificación a la herramienta virtual como la percepción general que tienen los estudiantes de la herramienta presentan un número considerable de estudiantes en el nivel de la indiferencia, es recomendable estudiar el motivo por el que se presenta este resultado

Se presentan diferencias notables en la percepción del uso de la herramienta por parte de los estudiantes en los tres centros universitarios

La información obtenida parece señalar que existe una subutilización de los Entorno Virtual de Aprendizaje dentro de las tres universidades.

En consecuencia, con lo anterior la evidencia parece mostrar que, a pesar de estar siendo subutilizado el EVA en las diferentes universidades, se tiene una impresión positiva por parte de los estudiantes en su uso.

Se destaca la importancia de trabajar proyectos conjuntos de nivel internacional aprovechando las redes de contactos, con la finalidad de realizar investigaciones orientadas a fortalecer el proceso de enseñanza aprendizaje, en este caso, relacionado a las plataformas virtuales en el ámbito universitario. 
A través del estudio realizado se evidenció que los estudiantes de Ecuador y Costa Rica utilizan en un alto porcentaje el aula virtual como herramienta de estudio para subir archivos y tareas en clase y extraclase, y los estudiantes de México para la participación en los foros, de esa manera se evidencia el uso de la herramienta tecnológico en las universidades participantes.

Las plataformas virtuales están siendo subutilizadas, para lo cual es conveniente desarrollar una estrategia de trabajo que suscite el uso de la mayoría de sus componentes en los períodos académicos, es aquí donde se establece una nueva pregunta de investigación y la posible solución a la misma.

\section{REFERENCIAS CONSULTADAS}

1. Baños, S. J. (2004). Entorno visual de aprendizaje.

2. Bautista, G. B. (2006). Didáctica universitaria en entornos virtuales de enseñanza aprendizaje. Madrid: Editorial Narcea.

3. Barrientos Oradini, N., Araya Castillo, L., Herrera Ciudad, F., \& Muñoz Morales, N. (2019). Actitud docente y apropiación tecnológica en educadores de nivel medio de la región metropolitana de Chile. Revista Arbitrada Interdisciplinaria Koinonía, 4(7), 33-58. doi:http://dx.doi.org/10.35381/r.k.v4i7.193

4. Belloch, C. (s.f.). Entornos Virtuales de Aprendizaje. Unidad de Tecnología Educativa (UTE). Recuperado el Enero de 2018, de Universidad de Valencia.

5. Belloch, C. (s.f.). Entornos virtuales de aprendizaje. Universidad de Valencia.

6. Boneu, J. (2007). Plataformas abiertas de e-learning para el soporte de contenidos educativos. Revista de Universidad y Sociedad del Conocimiento, 36-47.

7. Casales, R., Rojas, J., \& Paulí, G. (2008). Algunas experiencias didácticas en el entorno de la plataforma Moodle. Revista de informática educativa y medios audiovisuales. 
8. Correa, J. (2005). La integración de plataformas de e-learning en la docencia universitaria: Enseñanza, aprendizaje e investigación con Moodle en la formación inicial del profesorado, Revista Latinoamericana de Tecnología.

9. Delgado, M. \&. (2009). ESTRATEGIAS DIDÁCTICAS CREATIVAS EN ENTORNOS VIRTUALES PARA EL APRENDIZAJE. Revista Electrónica "Actualidades Investigativas en Educación".

10. Ferreiro, F. (s.f.). El constructivismo social.

11.Gimeno Sacristán, P. G. (1996). Comprender y transformar la enseñanza. Madrid: Morata.

12.Guitert, M., \& Romeu, T. y. (2017). Competencias TIC y trabajo en equipo en entornos virtuales. Recuperado el Enero de 2018, de Revista de Universidad y Sociedad del Conocimiento (RUSC). Vol. 4, n. ${ }^{\circ} 1$. UOC

13.Lattá Arias, C. (2019). Uso de las TIC para proyectos productivos en las instituciones educativas del Municipio Zona Bananera. Magdalena. Colombia. Revista Arbitrada Interdisciplinaria Koinonía, 4(7), 233-246. doi:http://dx.doi.org/10.35381/r.k.v4i7.202

14.Moodle (2018). Estadísticas Moodle. Recuperado de https://docs.moodle.org/all/es/Estad\%C3\%ADsticas

15. Onrubia, J. (s.f.). Aprender y enseñar en entornos virtuales: actividad. RED. Revista de Educación a Distancia, número monográfico II.

16. Ordóñez, C. L. (2004). Pensar pedagógicamente desde el constructivismo. De las concepciones a las prácticas pedagógicas. Revista de Estudios Sociales.

17.Orquera, M. (2012). Aulas Virtuales en la Educación Presencial.

18. Ros, I. (2008). Moodle, la plataforma para la enseñanza y organización escolar. Obtenido de Ikastorratza, e- Revista de Didáctica 2

19.Salinas, J. (2004). Cambios metodológicos con las TIC. Estrategias didácticas y entornos virtuales de enseñanza-aprendizaje.

20.Salmerón, H. \&. (2010). Metodologías que optimizan la comunicación en entornos de aprendizaje virtual. Comunicar, XVII (34), 163-171.

21.Silva, Q. J. (2011). Diseño y moderación de entornos virtuales de aprendizaje (EVA). Barcelona: UOC. 
22. Tenutto, M., Klinoff, A., \& Boan. (2005). Escuela para maestros. Colombia: Printer colombiana S.A.

23. Umanzor, P. (s.f). El enfoque constructivista como estrategia para mejorar la calidad de la educación.

24. Urueta Vélez, L. (2019). Estrategias de enseñanza y el uso de las tecnologías de información y comunicación en las instituciones educativas departamentales en el Municipio Zona Bananera - Colombia. Revista Arbitrada Interdisciplinaria Koinonía, 4(7), 185-201. doi:http://dx.doi.org/10.35381/r.k.v4i7.200

25. Vega Gil, L. (2010). El proceso de Bolonia y la educación comparada. Miradas críticas.

\section{REFERENCES CONSULTED}

1. Baños, S. J. (2004). Visual learning environment.

2. Bautista, G. B. (2006). University teaching in virtual learning teaching environments. Madrid: Narcea Publishing House.

3. Barrientos Oradini, N., Araya Castillo, L., Herrera Ciudad, F., \& Muñoz Morales, N. (2019). Teaching attitude and technological appropriation in middle level educators in the metropolitan region of Chile. Interdisciplinary Arbitrated Review Koinonía, 4 (7), 33-58. doi: http: //dx.doi.org/10.35381/r.k.v4i7.193

4. Belloch, C. (s.f.). Virtual Learning Environments. Educational Technology Unit (UTE). Retrieved on January 2018, from the University of Valencia.

5. Belloch, C. (s.f.). Virtual learning environments. University of Valencia

6. Boneu, J. (2007). Open e-learning platforms to support educational content. University and Knowledge Society Magazine, 36-47.

7. Casales, R., Rojas, J., \& Paulí, G. (2008). Some educational experiences in the environment of the Moodle platform. Journal of educational informatics and audiovisual media.

8. Correa, J. (2005). The integration of e-learning platforms in university teaching: Teaching, learning and research with Moodle in initial teacher training, Latin American Journal of Technology. 
9. Delgado, M. \&. (2009). CREATIVE DIDACTIC STRATEGIES IN VIRTUAL ENVIRONMENTS FOR LEARNING. Electronic Magazine "Investigative News in Education".

10.Ferreiro, F. (s.f.). The social constructivism.

11.Gimeno Sacristán, P. G. (1996). Understand and transform teaching. Madrid: Morata.

12.Guitert, M., \& Romeu, T. and. (2017). ICT skills and teamwork in virtual environments. Retrieved on January 2018, from University and Knowledge Society Magazine (RUSC). Vol. 4, \# 1.UOC

13.Lattá Arias, C. (2019). Use of ICT for productive projects in the educational institutions of the Municipality of Banana Zone. Cupcake Colombia. Interdisciplinary Arbitrated Review Koinonía, 4 (7), 233-246. doi: http: //dx.doi.org/10.35381/r.k.v4i7.202

14.Moodle (2018). Moodle statistics. Recovered from https://docs.moodle.org/all/es/Estad\%C3\%ADsticas

15. Onrubia, J. (s.f.). Learn and teach in virtual environments: activity. NET. Distance Education Magazine, monographic number II.

16.Ordóñez, C. L. (2004). Think pedagogically from constructivism. From conceptions to pedagogical practices. Social Studies Magazine.

17. Orquera, M. (2012). Virtual Classrooms in Face-to-face Education.

18. Ros, I. (2008). Moodle, the platform for teaching and school organization. Obtained from Ikastorratza, e- Journal of Didactics 2

19.Salinas, J. (2004). Methodological changes with ICT. Teaching strategies and virtual teaching-learning environments.

20.Salmerón, H. \&. (2010). Methodologies that optimize communication in virtual learning environments. Communicate, XVII (34), 163-171.

21.Silva, Q. J. (2011). Design and moderation of virtual learning environments (EVA). Barcelona: UOC.

22.Tenutto, M., Klinoff, A., \& Boan. (2005). School for teachers. Colombia: Printer colombiana S.A. 


\section{CIENCIAMATRIA}

Revista Interdisciplinaria de Humanidades, Educación, Ciencia y Tecnología

Año VI. Vol. VI. №10. Enero - Julio 2020

Hecho el depósito de ley: pp201602FA4721

ISSN-L: 2542-3029; ISSN: 2610-802X

Universidad Nacional Experimental Francisco de Miranda (UNEFM). Santa Ana de Coro. Venezuela

Irma Abrigo Cordoba; Donald Granados Gómez; Nancy Sánchez Sulú; Yolanda Margarita Celi Vivanco

23. Umanzor, P. (s.f). The constructivist approach as a strategy to improve the quality of education.

24. Urueta Vélez, L. (2019). Teaching strategies and the use of information and communication technologies in departmental educational institutions in the Municipality of Bananera Zone - Colombia. Interdisciplinary Arbitrated Review Koinonía, 4 (7), 185-201. doi: http: //dx.doi.org/10.35381/r.k.v4i7.200

25. Vega Gil, L. (2010). The Bologna process and comparative education. Critical looks. 